\title{
UN MODÈLE 3D DES “PERTURBATIONS” DES SÉRIES PHOSPHATÉES BASÉ SUR L'OPTIMISATION DES RÉPONSES DU SIGNAL ANALYTIQUE DES DONNÉES DE RÉSISTIVITÉ ÉLECTRIQUE
}

\begin{abstract}
Bakkali, S. et Amrani, M.
Faculty of Sciences \& Techniques, University Abdelmalek Essaâdi, BP 416, Tangier, Morocco

RÉSUMÉ: Une campagne de prospection électrique de type Schlumberger a été réalisée sur une zone couvrant une surface $50 \mathrm{Ha}$ dans une bassin phosphatier. Un nouveau procédé basé sur l'optimisation des réponses du signal analytique des données de résistivité a été réalisé afin de circonscrire les zones anomales de "perturbations » des séries phosphatées. Des modèles de terrain $3 D$ ont été obtenus le long d'une profondeur de $30 \mathrm{~m}$. Ces procédés ont permis de caractériser spatialement les anomalies géoélectriques tout en amoindrissant leurs caractères aléatoires. Les estimations de calcul des réserves se trouvent fortement circonscrites.
\end{abstract}

Mots Clefs: résistivité, phosphates, modélisation, signal analytique, prolongement, Maroc

ABSTRACT: A Schlumberger resistivity survey over an area of 50 hectares was carried out. A new field procedure based on analytic signal response of resistivity data was tested to deal with the presence of phosphate deposit disturbances. Models of the geology were successfully obtained from surface modeling of $2 D$ peaks of resistivity data. Image processing optimization was based on surface optimization tools. Downward analytical prolongation of the surface modeling along 30 meters of depth was used for modeling optimization. Analytical procedures were found to be consistently useful. Optimization of phosphate reserves were improved and better constrained.

Key words : resistivity, phosphate, surface modeling, analytic signal, downward, Morocco

\section{INTRODUCTION}

Avec une capacité de production de 19 millions de tonnes par an, et plus de 35 milliards de $\mathrm{m}^{3}$ de réserves, le Maroc détient actuellement plus de $75 \%$ des réserves mondiales des phosphates. Dans ce contexte spécifiquement industriel, la prospection géophysique par la méthode des résistivités électriques a été appliquée dans le bassin phosphatier Oulad Abdoun dans la province de Khouribga (figure 1) située à près de $120 \mathrm{~km}$ au sud est de la capitale économique Casablanca.

L'étude géophysique avait pour cadre spécifique le gisement de Sidi Chennane, l'une des parcelles du bassin sédimentaire Oulad Abdoun. Le gisement de phosphate est de type sédimentaire, présentant plusieurs couches phosphatées. Les couches phosphatées alternent avec des niveaux de marnes et de calcaires. Le gisement de Sidi Chennane a été retenu pour prendre la relève du gisement dit du Grand Daoui. Au sein du gisement des discontinuités appelées «perturbations » affectent totalement et/ou partiellement la succession habituelle de la série phosphatée. Stériles, généralement très durs et détectables uniquement lors de la foration, les « perturbations » perturbent les chaînes cinématiques d'exploitation en biaisant les calculs de réserve. Les méthodes directes telles les coupes de puits et les indices de surface s'étaient avérées insuffisantes et non adaptées pour la reconnaissance de ces discontinuités. La résistivité de la série phosphatée normale est comprise entre 80 et $150 \Omega$.m; celle des « perturbations » est comprise entre 200 et plus de $1000 \Omega$.m. Dans ce contexte particulier une étude géophysique pilote de prospection électrique en courant continu avait été réalisée. Couvrant un panneau de $50 \mathrm{Ha}$, cette étude pilote avait pour objectif de cartographier et de délimiter en surface les zones anomales correspondant aux «perturbations ». La carte de résistivité fournit une interprétation directe et imagée des séries de profils de traînées électriques dont elle découle. Dans notre cas une carte de résistivité apparente fut réalisée. Le dispositif utilisé fut un dispositif Schlumberger centré de 


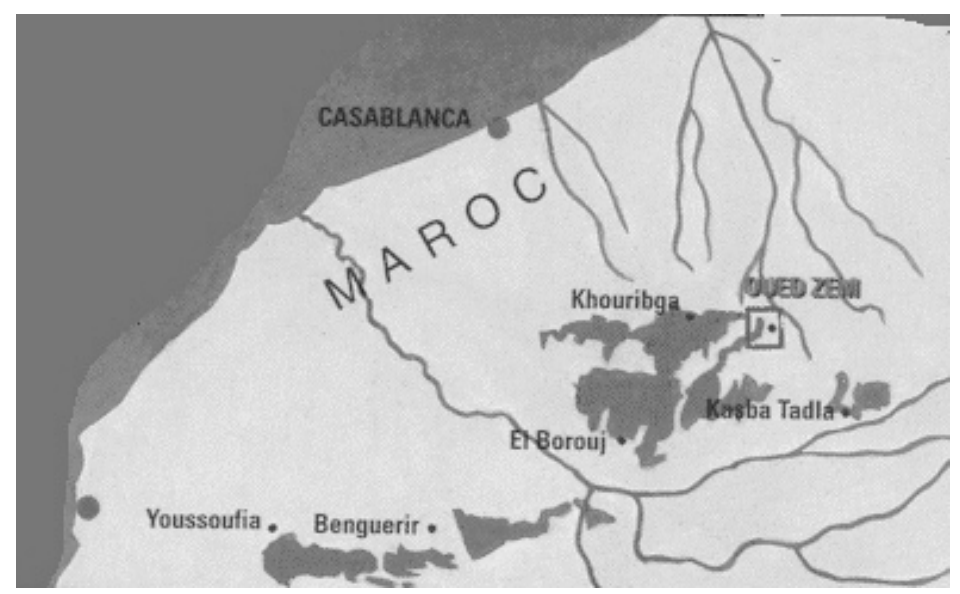

Figure 1: carte de situation géographique de la zone d'étude

longueur de ligne $A B=120 \mathrm{~m}$. Ce procédé avait permis d'identifier les zones anomales coïncidant avec les «perturbations » pour des profondeurs moyennes inférieures à $40 \mathrm{~m}$. Les « perturbations » se présentent de façon aléatoire. La carte joue le rôle fondamental de « radar » pour les conducteurs de travaux, et ce afin de ne pas briser la chaîne cinématique d'exploitation. Les techniques classiques du filtrage spatial servent alors à circonscrire efficacement les effets aléatoires de la distribution des «perturbations » correspondant aux anomalies géoélectriques. L'objetif de cette étude consiste en une modélisation 3D des "perturbations » par optimisation des réponses su signal analytique des résistivités apparentes. Cette méthodologie permettra aux conducteurs de travaux d'exploiter adéquatement les séries phosphatées saines et non «perturbées ».

L'étude géophysique avait pour cadre spécifique le gisement de Sidi Chennane, l'une des parcelles du bassin sédimentaire Oulad Abdoun. Le gisement de phosphate est de type sédimentaire, présentant plusieurs couches phosphatées. Les couches phosphatées alternent avec des niveaux de marnes et de calcaires. Le gisement de Sidi Chennane a été retenu pour prendre la relève du gisement dit du Grand Daoui. Au sein du gisement des discontinuités appelées "perturbations » affectent totalement et/ou partiellement la succession habituelle de la série phosphatée. Stériles, généralement très durs et détectables uniquement lors de la foration, les «perturbations » perturbent les chaînes cinématiques d'exploitation en biaisant les calculs de réserve. Les méthodes directes telles les coupes de puits et les indices de surface s'étaient avérées insuffisantes et non adaptées pour la reconnaissance de ces discontinuités. La résistivité de la série phosphatée normale est comprise entre 80 et $150 \Omega . \mathrm{m}$; celle des « perturbations » est comprise entre 200 et plus de $1000 \Omega . m$. Dans ce contexte particulier une étude géophysique pilote de prospection électrique en courant continu avait été réalisée. Couvrant un panneau de $50 \mathrm{Ha}$, cette étude pilote avait pour objectif de cartographier et de délimiter en surface les zones anomales correspondant aux « perturbations ». La carte de résistivité fournit une interprétation directe et imagée des séries de profils de traînées électriques dont elle découle. Dans notre cas une carte de résistivité apparente fut réalisée. Le dispositif utilisé fut un dispositif Schlumberger centré de longueur de ligne $\mathrm{AB}=120 \mathrm{~m}$. Ce procédé avait permis d'identifier les zones anomales coüncidant avec les «perturbations» pour des profondeurs moyennes inférieures à $40 \mathrm{~m}$. Les « perturbations » se présentent de façon aléatoire. La carte joue le rôle fondamental de « radar » pour les conducteurs de travaux, et ce afin de ne pas briser la chaîne cinématique d'exploitation. Les techniques classiques du filtrage spatial servent alors à circonscrire efficacement les effets aléatoires de la distribution des «perturbations » correspondant aux anomalies géoélectriques. L'objetif de cette étude consiste en une modélisation 3D des «perturbations » par optimisation des réponses su signal analytique des résistivités apparentes. Cette méthodologie permettra aux conducteurs de travaux d'exploiter adéquatement les séries phosphatées saines et non "perturbées ».

\section{Présentation géographique de la zone d'étude}

Le gisement de Sidi Chennane du bassin phosphatier Oulad Abdoun est localisé à $33 \mathrm{~km}$ au sud est de Khouribga 
Un modèle 3D des "perturbations" des séries phosphatées basé sur l'optimisation des réponses du signal analytique des données de résistivité électrique

(figure 2). Il est délimité à l'ouest par le méridien Lambert 372500 au sud par le méridien 228000, à l'est par la route principale RP22 et au nord par les affleurements de la base de la série phosphatée. Le plateau des phosphates est essentiellement une région à climat aride. La pluviométrie, étalée sur les mois allant de novembre à mai, ne dépasse généralement guère les $400 \mathrm{~mm}$, ne permettant ainsi qu'une végétation clairsemée principalement représentée par des palmiers nains. La population rurale, vivant particulièrement d'élevage et de cultures saisonnières est dispersée dans de petits villages ou douars. Les besoins en eau de cette région sont de plus en plus pressants, et les manques y sont surtout enregistrés avec une certaine gravité. Exceptés quelques puits aléatoirement éparpillés alimentés par les infiltrations au cours de la période hivernale, et dont les marnes sénoniennes forment l'écran imperméable, le seul réservoir aquifère est représenté par le calcaire turonien. Ce dernier situé généralement à plus de $100 \mathrm{~m}$ de profondeur, constitue la roche réservoir la plus importante de la région, jouant ainsi un rôle déterminant dans l'alimentation en eau de divers centres d'exploitation.

\section{Contexte géologique}

Les phosphates ont été déposés sur une longue période allant de la fin du Crétacé (étage du Maestrichtien, environ
80 Millions d'années) jusqu'au début de l'Eocène (étage du Lutétien basal ou Lutétien inférieur 40 Millions d'années). Néanmoins entre ces périodes les dépôts n'étaient pas constants. Il manque par conséquent des sédiments: ce qui atteste de la discontinuité de la chaîne d'information géologique. Le bassin des Oulad Abdoun couvre la partie principale de l'élément morphologique appelé plateau des phosphates. Il est borné au nord par les affleurements des dépôts rouges infracénomaniens qui prolongent le flanc sud du massif central, à l'ouest par le massif des Rhamna, au Sud par la plaine de Beni Amir et à l'est par le haut Atlas de Béni Mellal. Géologiquement, la série de ce plateau des phosphates auquel est identifiée la région de notre site d'étude est bien étudiée.

Les «perturbations » objet de cette étude associent les paramètres tels l'ampleur, la nature des matériaux, la dureté, la teneur en argile, la nature du contact « perturbation »série normale. Les « perturbations » ont été singulièrement différenciées selon 2 types prédominants. Le premier type affecte toute la série phosphatée correspond aux «perturbations » formées d'un mélange hétéroclite de calcaires, de marnes, d'argiles, de silex et phosphate avec abondance de calcaire riche en silex. Le second type représente une série totalement perturbée sans prédominance de lithofaciès. La «perturbation» se présente sous forme d'un amas de blocs de calcaires

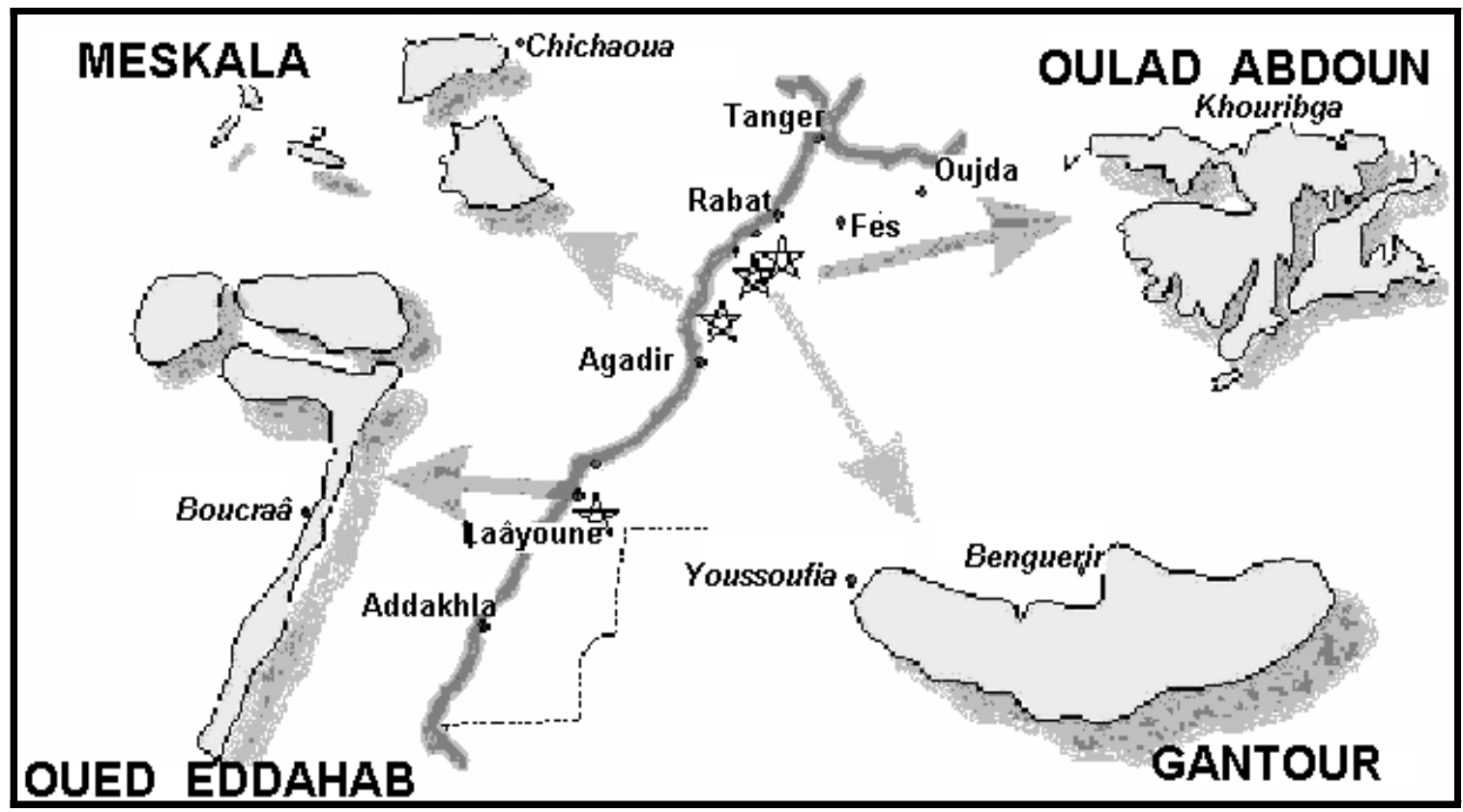

Figure 2: carte des bassins des phosphates du Maroc 
phosphatés à gros nodules de silex, des marnes, des argiles, des portions de bancs de silex et des phosphates (Kchikach et al., 2002). Ce type de «perturbation» dont les dimensions ont des diamètres variant de 10 à plus de $150 \mathrm{~m}$, est le plus fréquemment rencontré lors de l'exploitation (figure 3).
Il affecte le primaire stérile et les termes supérieurs de la série phosphatée. Ces particularités se traduisent par un fort contraste de résistivité entre les «perturbations » et la série phosphatée normale telle que confirmée par les profils-étalon de résistivité (figure 4). La campagne de prospection géophysique a été ainsi basée sur la mise en

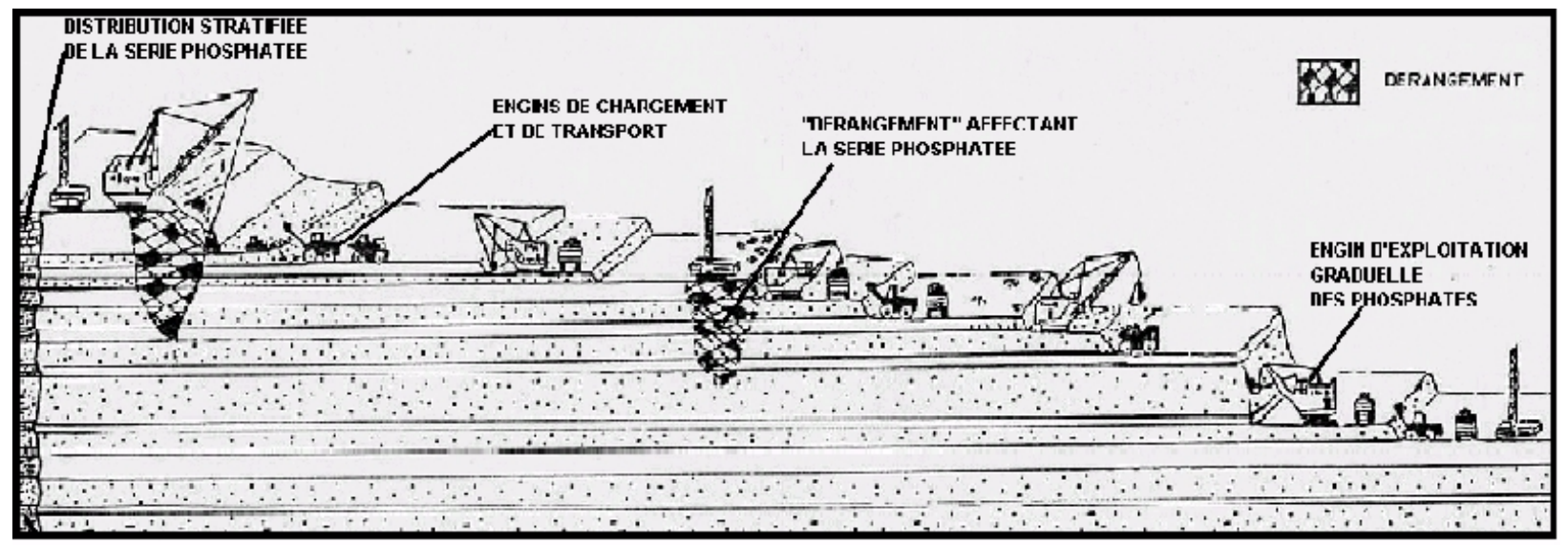

Figure 3: Impact des "perturbations " sur la chaîne d'exploitation

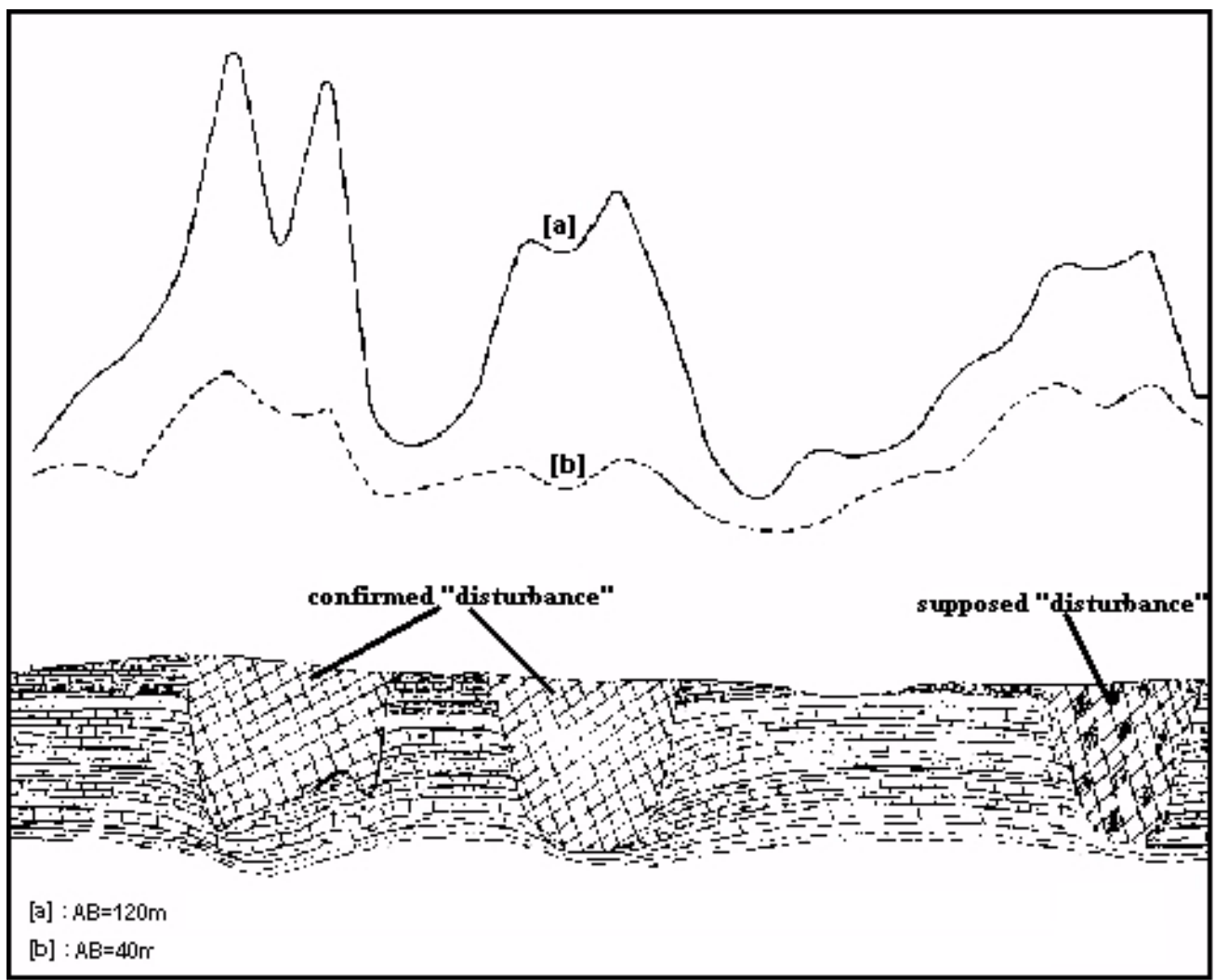

Figure 4: profils de résistivités sur "perturbations" 
Un modèle 3D des "perturbations" des séries phosphatées basé sur l'optimisation des réponses du signal analytique des données de résistivité électrique

évidence de ces contrastes singuliers de résistivité sur l'ensemble du panneau de cette étude pilote.

\section{MATÉRIELETMÉTHODE}

La méthode du trainée électrique a été utilisée pour délimiter les zones anomales correspondantes aux «perturbations ». Le principe général de la prospection électrique par trainés électriques repose sur l'injection d'un courant électrique I entre deux électrodes A et B (dites de courant) et la mesure d'une différence de potentiel $\Delta \mathrm{V}$ entre deux autres électrodes $\mathrm{M}$ et $\mathrm{N}$ (dites de potentiel). On en déduit la résistivité apparente du soussol: $\quad \rho_{\text {apparent }}=K \frac{\Delta V}{I}$ ou $\mathrm{K}$ est le coefficient géométrique du dispositif, fonction uniquement des distances entre électrodes (Bakkali et al., 2005). Le rapport des deux paramètres et permet de calculer la résistivité des terrains sous-jacents. Le dispositif Schlumberger utilisé dans ce travail nécessite que les électrodes soient alignées, symétriques par rapport à leurs centre $\mathrm{O}$ avec
$\mathrm{MN}<<\mathrm{AB}$. Les traînées électriques permettent d'obtenir des profils et des cartes de résistivités apparentes. Plus la longueur de ligne sera grande, plus la profondeur d'investigation augmentera (Bakkali et al., 2006). Les traînées électriques de résistivité apparente rendent compte de la variabilité horizontale des propriétés du soussol. Ils servent à déterminer l'extension surfacique des couches en une position donnée, et permettent de confirmer la présence ou l'absence des « perturbations ». Ils permettent également de circonscrire les variations latérales de faciès. Les mesures de résistivité apparente ont été réalisées avec le résistivimètre Syscal2 de BrgmInstruments aux sommets d'une maille rectangulaire de dimensions $20 \mathrm{~m}$ x $5 \mathrm{~m}$. Pour couvrir l'ensemble de la zone d'étude et afin de cibler des profondeurs de « perturbations » moyennes de $40 \mathrm{~m}, 51$ profils de traînées électriques régulièrement espacés de $20 \mathrm{~m}$ ont été exécutés. Chacun des profils électriques représente 101 stations de mesure équidistantes de $5 \mathrm{~m}$. L'équidistance entre les profils est de $20 \mathrm{~m}$. L'ensemble de cette étude pilote avait représenté ainsi plus de 5151 mesures de résistivité apparente (figure 5) (Bakkali, 2006).

DIRECTION NORD (DISTANCE EN METRES)

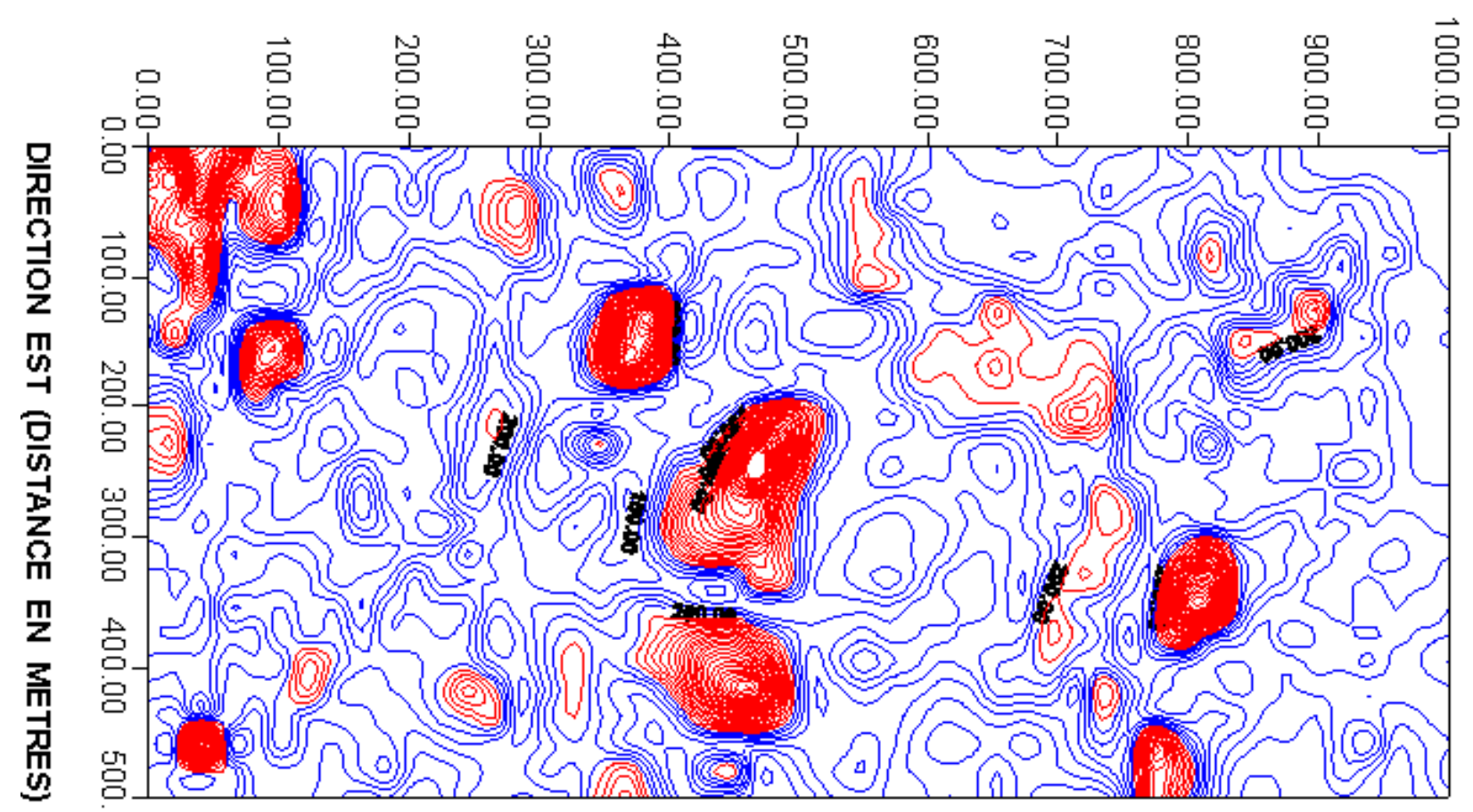

Ф̣̊igure 5: carte des résistivités apparentes de la zone d'étude (intervalle contour: $20 \Omega$.m). Les zones de "perturbations" correspondent aux résistivités anomales (iso-courbes rouges de résistivité $\geq 200 \Omega$.m) 


\section{Méthode du signal analytique}

L'interprétation directe des caractéristiques d'une anomalie géoélectrique permet d'obtenir rapidement différentes informations qualitatives et semi-quantitatives sur la nature et la position du corps perturbateur. Le corps perturbateur est supposé constitué essentiellement de «perturbations ». Les « perturbations » sont les corps potentiants. L'amplitude est proportionnelle au volume du corps perturbateur et au contraste de résistivité entre celui-ci et les roches encaissantes. Une structure n'apparaîtra pas si les roches qui la constituent ont la même résistivité que le terrain encaissant. La résistivité apparente découle d'un potentiel harmonique $\mathrm{V}$ vérifiant l'équation de Laplace $\Delta \mathrm{V}=0$ en dehors des corps potentiants, et dont le gradient de celui-ci est du type $1 / \mathrm{r}^{2}$ (Blakely, 1995). En première approche, il est supposé qu'au «voisinage» ainsi que dans les sources représentées par les « perturbations » le potentiel scalaire vérifie l'équation $\Delta V=-2 \rho I \delta(r)$ ou $\delta($ r) est la distribution de Dirac, $\rho$ est la résistivité de la zone anomale et I est le courant injecté par une électrode ponctuelle sur un demi-espace élastique (surface topographique de la zone d'étude) (Telford et al., 1995). La cartographie géoélectrique réalisée par mesures de résistivités apparentes sur une grille régulière est en fait une cartographie de potentiels discrets mesurés en surface. La singularité des fortes valeurs de résistivités apparentes sur les zones de "perturbations » résulte à juste titre du passage d'une zone saine à une zone " perturbée ». Sous ces hypothèses, la carte de résistivité apparente est en fait par analogie une carte de différences de potentiel scalaires supposés partout harmoniques excepté dans les zones «perturbées ». La carte des résistivités anomales permet une définition physique des sources. La carte de différences de potentiel s'adonne ainsi usuellement au traitement numérique des données dans le domaine spatial.

L'interprétation des anomalies de résistivité correspond au processus de $l^{\prime}$ extraction de l'information relative à la position et à la composition du corps potentiant. Dans notre cas les corps «potentiants » correspondent essentiellement aux «perturbations ». L'amplitude de l'anomalie est considérée proportionnelle au volume des « perturbations » ainsi qu'au contraste de résistivité existant entre une zone saine et une zone "perturbée ». Ce contraste de résistivité est proportionnel au contraste local de densité existant entre les zones phosphatées saines et les zones «perturbées ». Cette approche quantitative dans l'interprétation des anomalies de résistivité nous permet de considérer que le niveau de "perturbations» des séries phosphatées est proportionnel à l'amplitude des résistivités apparentes mesurées (Bakkali, 2005).

Les dérivées horizontale et verticale des anomalies de résistivité produites par une source potentielle forment une paire de transformée de Hilbert et définissent un signal analytique (Nabighian, 1972). La méthode du signal analytique, également définie comme étant la méthode du gradient total, est utilisée pour circonscrire les contours des anomalies de densité selon les directions orthogonales. La propriété importante du signal 3D analytique réside dans le fait que ses amplitudes locales forment l'enveloppe analytique de son signal fondamental (Kanasewich, 1981). L'intensité du gradient des données de résistivité est équivalente à l'enveloppe des gradients locaux horizontal et vertical selon toutes les directions possibles. L'amplitude du signal analytique est remarquable parce qu'elle permet d'obtenir un signal représentatif des anomalies de résistivité intégrant toutes les directions possibles relatives à la zone d'étude (figure 6).

Les maximas locaux de la carte du signal analytique correspondent fondamentalement aux zones anomales des «perturbations » de dépôt des séries phosphatées. Les maximas locaux du signal analytique ont la remarquable propriété de localiser directement les contacts singuliers correspondant aux anomalies de contraste local de densité. Ce processus analytique définit un filtrage spatial des zones anomales des « perturbations ».

\section{Expression de l'enveloppe surfacique du signal analytique}

L'amplitude globale en 3D du signal analytique appelée gradient total définit en fait une enveloppe surfacique.

Soit $A(\rho)$ le signal analytique de la résistivité apparente dans les directions Est et Nord. On suppose fonction de $x, y$ et $\vartheta$ et définie par l'expression suivante:

$$
A(\rho(x, y))=a(x, y) \cos \vartheta+b(x, y) \sin \vartheta
$$

ou $a(x, y)$ et $b(x, y)$ représentent les dérivées directionnelles respectivement dans les directions Est et Nord. $a(x, y)=\frac{\partial \rho(x, y)}{\partial x}$ et $b(x, y)=\frac{\partial \rho(x, y)}{\partial y}$. Le signal analytique $A(\rho)$ pourrait alors s'exprimer sous la 

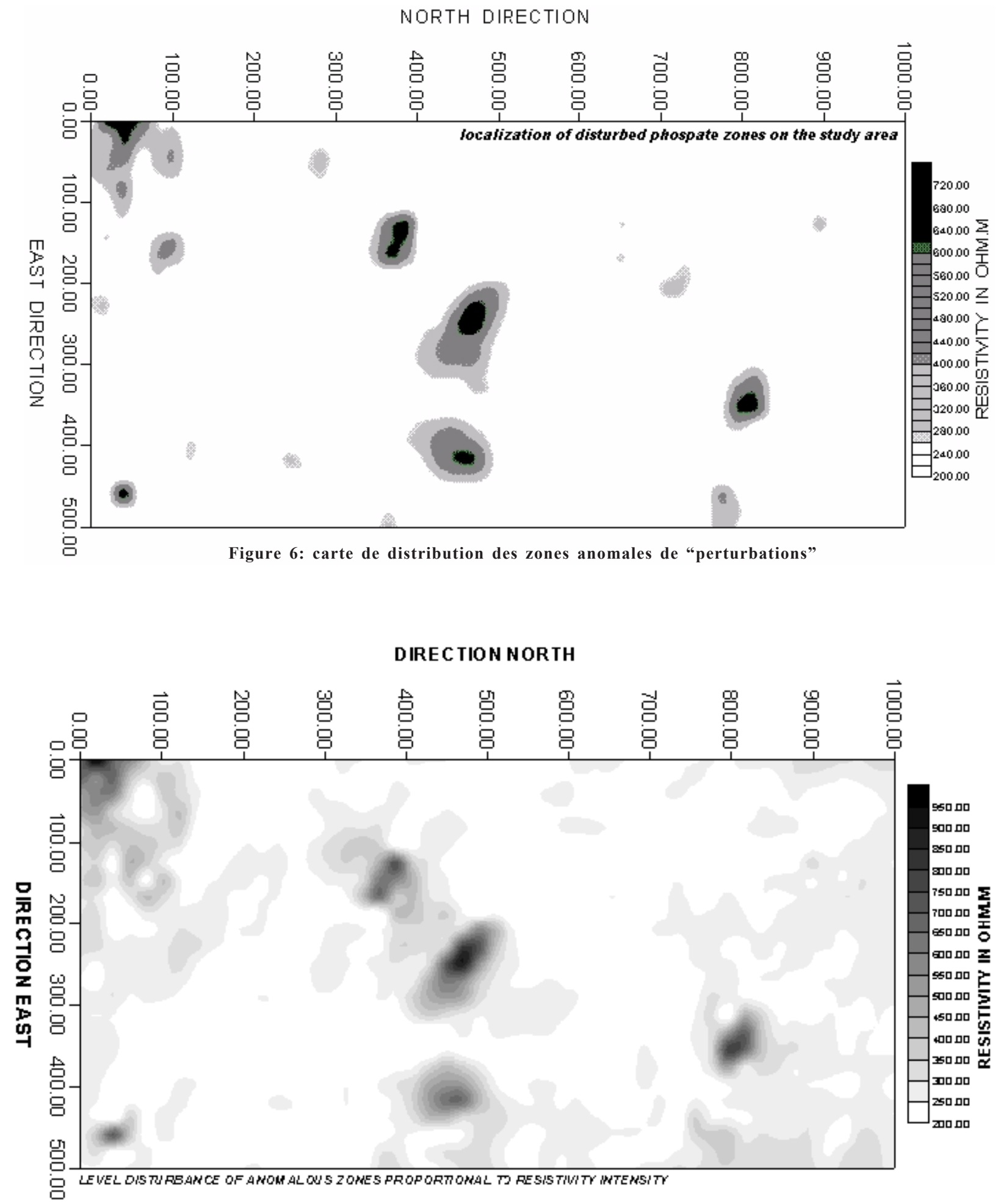

Figure 7: réponse du signal analytique de la figure 6 
forme suivante:

$A(\rho(x, y))=\frac{\partial \rho(x, y)}{\partial x} \cos \vartheta+\frac{\partial \rho(x, y)}{\partial y} \sin \vartheta$

ou $x$ et $y$ sont les coordonnées cartésiennes d'un point donné de la zone d'étude, $\vartheta$ étant l'angle définissant une direction quelconque.

L'enveloppe du signal analytique de la résistivité apparente intégrant toutes les valeurs de $\vartheta$, (cela permet de balayer l'ensemble de la zone d'étude sans direction privilégiée) peut être obtenue en utilisant les cosinus directeurs définis par (Zauderer, 1989):

$$
\begin{gathered}
\sin \vartheta=\frac{ \pm \frac{\partial \rho(x, y)}{\partial y}}{\sqrt{\left(\frac{\partial \rho(x, y)}{\partial x}\right)^{2}+\left(\frac{\partial \rho(x, y)}{\partial y}\right)^{2}}} \text { et } \\
\cos \vartheta=\frac{ \pm \frac{\partial \rho(x, y)}{\partial x}}{\sqrt{\left(\frac{\partial \rho(x, y)}{\partial x}\right)^{2}+\left(\frac{\partial \rho(x, y)}{\partial y}\right)^{2}}}
\end{gathered}
$$

En remplaçant les expressions des cosinus directeurs dans l'équation (1) on obtient l'expression de l'enveloppe du signal analytique de la résistivité apparente $\varepsilon|A(\rho(x, y))|$ définie par:

$\varepsilon|A(\rho(x, y))|=\sqrt{\left(\frac{\partial \rho(x, y)}{\partial x}\right)^{2}+\left(\frac{\partial \rho(x, y)}{\partial y}\right)^{2}}$

L'équation (4) peut également se mettre sous la forme

$\varepsilon|A(\rho)|=\sqrt{|\rho|^{2}+\left(\frac{\partial \rho}{\partial \vartheta}\right)^{2}}$

Les dérivées partielles sont calculées numériquement. Les gradients horizontaux sont usuellement estimés par la méthode des différences finies aux points d'une grille « griddée » représentant la carte des anomalies de résistivité correspondant à la carte des zones anomales des « perturbations » (Bakkali, 2005, 2006):

$$
\frac{\partial \rho(\mathrm{x}, \mathrm{y})}{\partial \mathrm{x}}=\frac{\rho_{\mathrm{i}+1, j}-\rho_{\mathrm{i}-1, \mathrm{j}}}{2 \delta \mathrm{x}} \text { et }
$$

$\frac{\partial \rho(\mathrm{x}, \mathrm{y})}{\partial \mathrm{y}}=\frac{\rho_{i, j+1}-\rho_{i, j-1}}{2 \delta \mathrm{y}}$ ou x et y représentent les

coordonnées dans les directions Est et Nord. $\rho_{i, j}$ est la pseudo-résistivité définie aux points $(i, j)$. Les pas d 'échantillonnage pour les calculs des dérivées partielles sont respectivement $\delta x$ et $\delta y$.

Les maximas de l'enveloppe surfacique du signal analytique des résistivités apparentes est alors donnée par l'équation suivante:

$(\varepsilon|A(\rho)|)_{i, j}=\sqrt{\left(\frac{\rho_{i+1, j}-\rho_{i-1, j}}{2 \delta x}\right)^{2}+\left(\frac{\rho_{i, j+1}-\rho_{i, j-1}}{2 \delta y}\right)^{2}}$

Nous avons procédé à l'interpolation des différents maximas du signal analytique en utilisant l'optimisation de l'enveloppe surfacique des résistivités apparentes correspondant aux zones anomales des «perturbations » (figure 8). L'optimisation de l'enveloppe surfacique est la propriété majeure développée par le logiciel TableCurve 3D (Systat, 2002) que nous avons utilisé.

La figure 8 représente un indicateur du degré de variation du contraste local de densité entre les zones saines des séries phosphatées et les zones des "perturbations" dites zones « dérangées ». La modélisation 3D de la surface des « perturbations » est obtenue à partir des algorithmes d'optimisation du logiciel TableCurve 3D. Ce procédé nous a permis de définir et de considérer les «perturbations » comme des sources potentielles, après un filtrage par les réponses du signal analytique. Ces procédures de modélisation permettent de connecter les zones surfaciques des «perturbations » à leurs sources en profondeur en considérant que leurs dimensions et profondeurs d'enfouissement sont proportionnelles aux maximas de la résistivité apparente de la zone d'étude. Le contraste local de densité entre les zones des «perturbations » et les zones saines non «perturbées » atteste que les premières sont fortement accentuées en surface. L'effet global résultant est équivalent à un scanner des corps anomaliques que sont les «perturbations » (figure 9). 


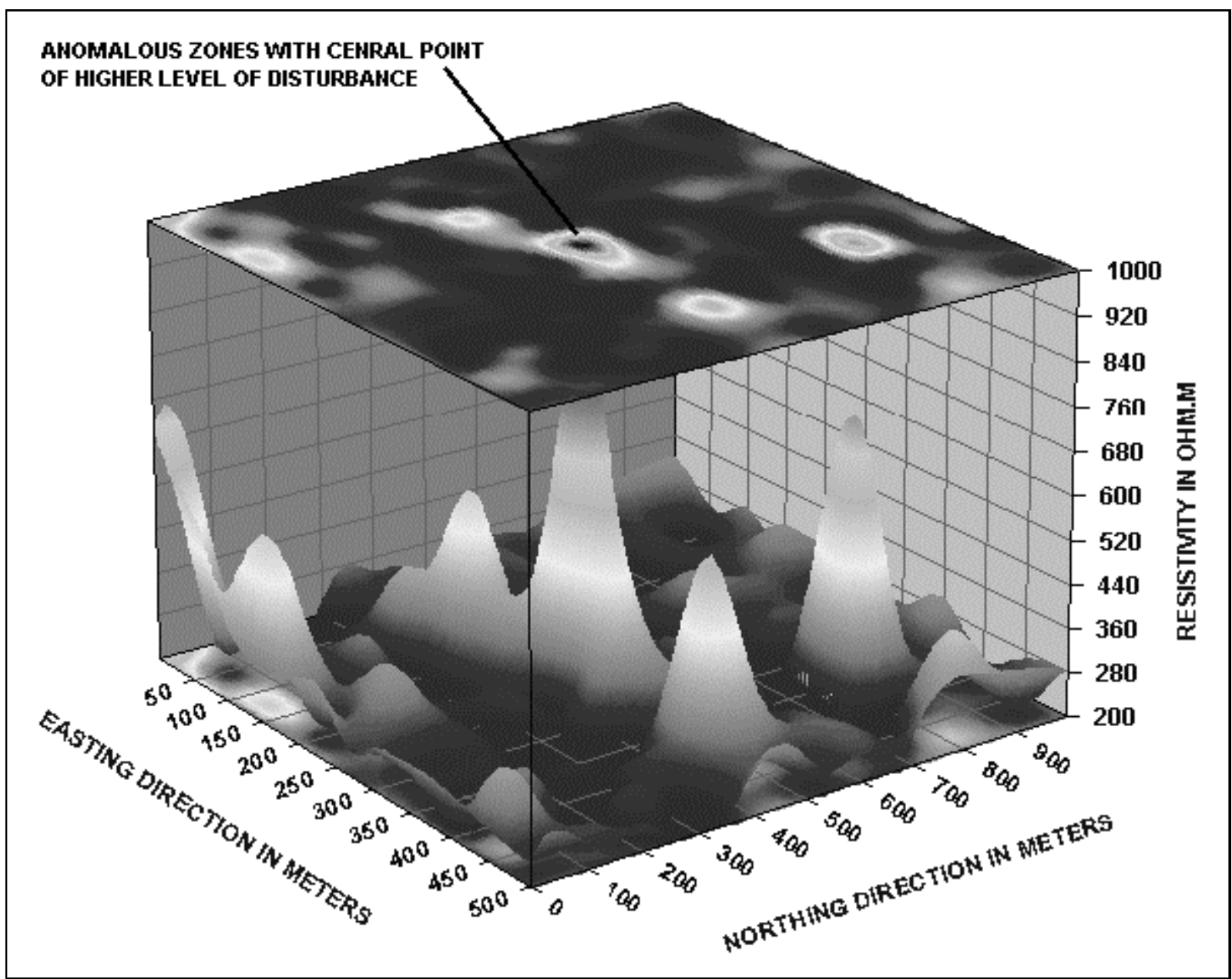

Figure 8: modèle de la surface des "perturbations" utilisant l'optimisation de la surface de l'enveloppe des maximas du signal analytique des résistivités apparentes.

Le prolongement analytique vers le bas permet d'amplifier l'effet des structures recherchées. Il permet de lever l'indétermination des anomalies coalescentes (Bakkali, 2006). Deux sources proches l'une de l'autre en profondeur fusionnent leurs anomalies en surface. Ces traitements, qui ne sont autres que des filtrages en terme de l'analyse de Fourier, suppriment certains caractères des anomalies et mettent en évidence d'autres.

Le prolongement analytique vers le bas permet d'augmenter le pouvoir séparateur de deux sources. Ce procédé permet dans une certaine mesure de cerner géométriquement la forme des structures anomales. Il permet le cas échéant de situer la profondeur du toit des structures anomales. Cependant il est possible que ce faisant plusieurs structures soient «traversées » (Lutz, 1999). Les données sont systématiquement symétrisées dans le domaine spatial afin de minimiser les effets de bord sur la carte transformée. L'opérateur de prolongement vers le bas peut être exprimé en domaine fréquentiel par $\mathrm{e}^{2 \pi v z}$ où $\mathrm{z}$ est la profondeur ciblée.. La fréquence $v$ peut être définie comme $v=\sqrt{ }\left(u^{2}+v^{2}\right)$, où $u$ et $v$ sont les fréquences respectivement dans les directions Est et Nord (Gunn, 1975). Le prolongement analytique de la carte des résistivités anomaliques a été opéré sur une profondeur totale de $30 \mathrm{~m}$ par pas successifs de $5 \mathrm{~m}$. Les cartes de résistivités prolongées, correspondantes aux cartes successives des «perturbations », ont permis de dresser des modèles géométriques de la surface des « perturbations » 


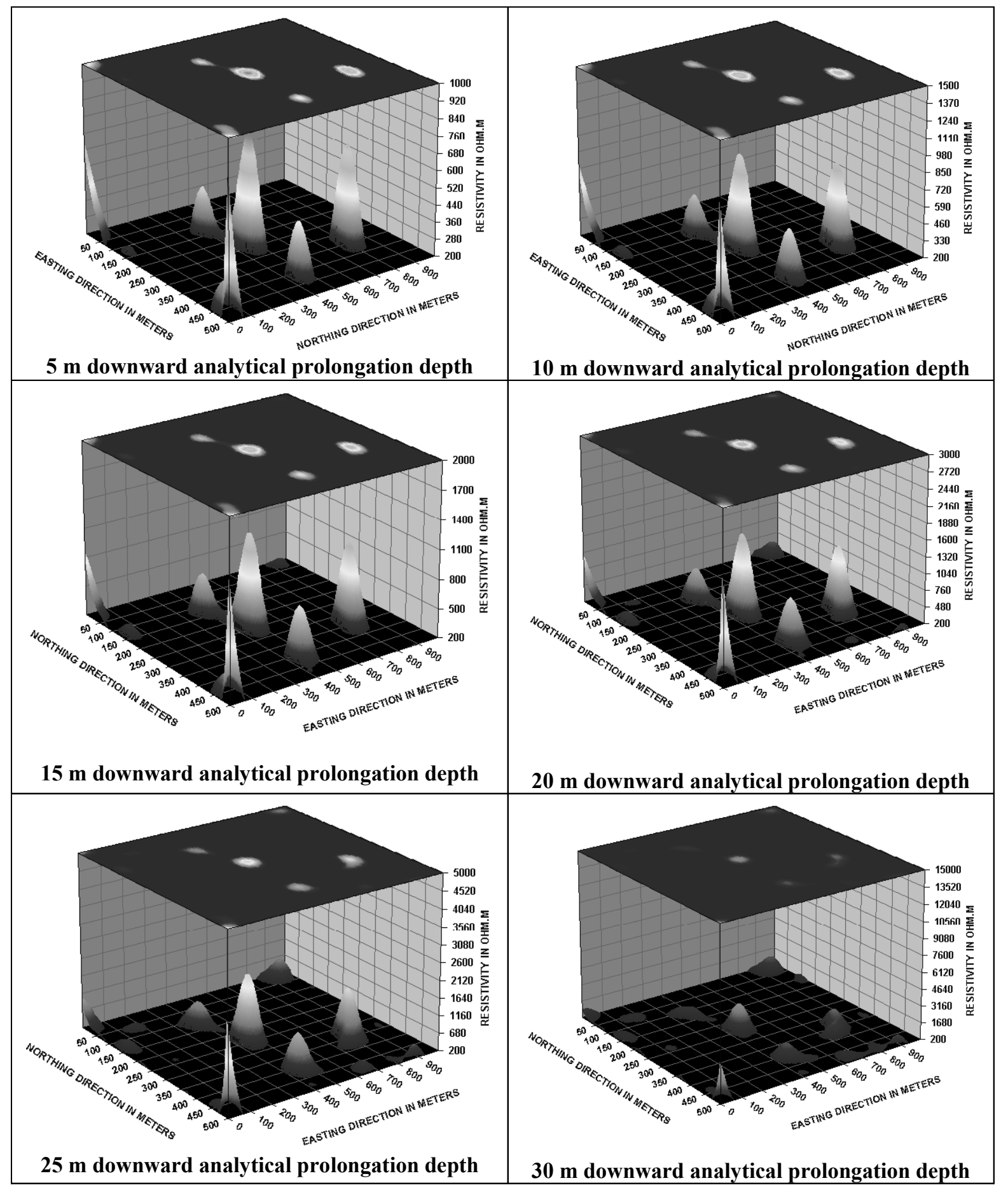

Figure 9: réponses analytiques du prolongement analytique vers le bas de la figure 8 à différentes profondeurs 
Un modèle 3D des "perturbations" des séries phosphatées basé sur l'optimisation des réponses du signal analytique des données de résistivité électrique

\section{RÉSULTATS}

Les enveloppes surfaciques de la carte des résistivités anomaliques obtenues à partir de ce procédé fournissent une image directe pour l'interprétation des données de résistivité issues de la prospection électrique. (figure 9). Les anomalies fortement corrélées avec les «perturbations » ont été identifiées. Les « perturbations » détectées en surface se présentent de façon aléatoire.

La carte des résistivités anomaliques correspondant aux zones anomales des «perturbations » a été « réhaussée » et filtrée par la méthode du signal analytique. Les zones anomales ont été bien différenciées. Les zones anomales des «perturbations » ont été précisées et mises en évidence sur une profondeur de $30 \mathrm{~m}$. Les valeurs élevées de la résistivité apparente des zones anomales indiquent que les « perturbations » sont consolidées en profondeur alors que leurs extensions s'amoindrissent. Les modèles de terrain obtenus par optimisation de l'enveloppe surfacique des «perturbations» le long des différentes profondeurs attestent ces particularités.

\section{CONCLUSIONS}

Cette étude pilote singulière a permis de donner une réponse analytique à l'analyse d'une carte de données géophysiques dans le domaine minier. La méthode géoélectrique utilisée dans cette approche originale a fourni des résultats satisfaisants tout en confirmant l'adaptation de la méthode des résistivités. Les zones anomales correspondant aux «perturbations» sont assimilées aux distributions anormales de densité. La méthode des réponses du signal analytique appliquée à la modélisation 3D des zones anomales des «perturbations » est jugée satisfaisante. Les différents modèles de terrain $3 \mathrm{D}$ obtenus par prolongement analytique vers le bas permettent d'optimiser les calculs des réserves des phosphates «utiles». Ils permettent également aux géomètres-topographes de circonscrire efficacement les surfaces anomales des réserves des phosphates.

\section{RÉFÉRENCES}

Bakkali, S. and L. Bahi, 2005. Cartographie des « dérangements » de séries phosphatées par mesures de résistivités électriques, Journal des Sciences Pour l’Ingénieur, JSPI, Vol.6 pp1-10.
Blakely, R.J., 1995. Potential Theory in Gravity and Magnetic Applications, Cambridge University Press, pp 441.

Bakkali, S., 2006. Application du filtrage spatial à l'analyse des contours des zones anomales de «dérangements» des séries phosphatées de Sidi Chennane (Maroc), Revue Afrique Science, Vol.2, №2, (under press)

Bakkali, S., 2006. A resistivity survey of phosphate deposits containing hardpan pockets in Oulad Abdoun, Morocco, Geofisica Internacional, 45 (1), p73-82.

Bakkali, S. and L. Bahi, 2006. Cartographie des "dérangements » de séries phosphatées par mesures de résistivités électriques, Journal des Sciences Pour l'Ingénieur, J.S.P.I., 6, p1-10.

Bakkali, S. and J. Bouyalaoui., 2005. Essai d'optimisation de la capacité de retenue d'eau d'un lac par caractérisation géophysique du recouvrement argileux, African Journal of Science \& Technology, AJST, 6(2).

Bakkali, S., 2005. Analysis of phosphate deposit "disturbances" using the horizontal-gradient responses of resistivity data (Oulad Abdoun, Morocco), Earth Sci. Res. J. Vol.9, º2, p123-131.

Kanasewich, E.R.,1981, Time sequence analysis in Geophysics, The University of Alberta Press.

Kchikach, A., Jaffal, M., Aifa, T. and Bahi, L., 2002. Cartographie de corps stériles sous couverture quaternaire par méthode de résistivités électriques dans le gisement phosphaté de Sidi Chennane (Maroc). Comptes Rendus. Geosciences, 334, 379-386.

Nabighian, M.N., 1972. The analytic signal of two dimensionnal bodies with polygonal cross section: its properties and use for automated anomaly interpretation, Geophysics, 37, 507.

Systat, 2002, About TableCurve 3Dl software version 4.0, Manuel d'utilisation, Copyright Systat Software Inc, 1993-2002.

Telford, W. M. and R.E Sheriff, 1991. Applied Geophysics, Cambridge University Press, pp790.

Zauderer, E., 1989, Partial differential equations of applied mathematics, Wiley-Interscience. 